\title{
Elementary Methods of interpreting the Equation of the first degree in two and three dimensions.
}

The following elementary methods of interpreting the equation of the first degree in two and three dimensions may be of some pedagogic interest.

I. (i) The equation $l x+m y+n=0$.

Let $M \equiv(m, 0)$ and $L \equiv(0, l)$.

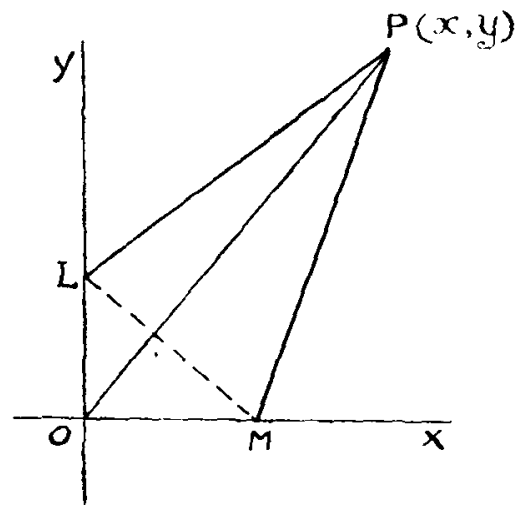

Let $P \equiv(x, y)$.

Then $l x=2 \triangle O P L$, and $m y=2 \triangle O P M$.

$\therefore \quad l x+m y=2$ quad. OLPM.

Hence if $P$ lies on the locus $l x+m y+n=0$, then

$$
\text { quad. } \begin{aligned}
O L P M & =-\frac{n}{2} \\
& =\text { constant area. }
\end{aligned}
$$

But $\triangle O L M$, which is part of the quadrilateral, is fixed, therefore $\triangle P L M$ is of constant area. The latter has a fixed base, so that the locus of the vertex is a unique (in virtue of the area of the quadrilateral) straight line parallel to $L M$.

Corollaries:

(a) Linear equations which differ only in the absolute terms are represented by parallel straight lines. 
ELEMENTARY METHODS OF INTERPRETING THE EQUATION, ETC.

( $\beta$ ) If the graphs of $l_{1} x+m_{1} y+n_{1}=0$ and $l_{2} x+m_{2} y+n_{2}=0$ are parallel straight lines, then $\frac{l_{1}}{l_{2}}=\frac{m_{1}}{m_{2}}$.

(ii) Length of the perpendicular from the point $P \equiv\left(x^{\prime}, y^{\prime}\right)$ to the straight line $l x+m y+n=0$.

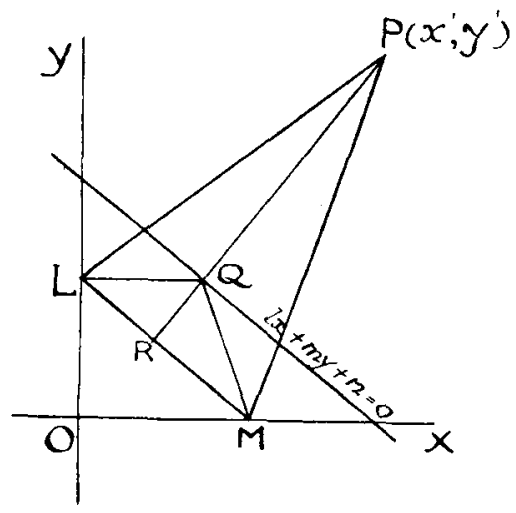

Let $M \equiv(m, 0)$ and $L \equiv(0, l)$.

Then $L M$ is parallel to $l x+m y+n=0$.

Draw $P Q R$ perpendicular to these parallels.

We have by (1) and (2) of (i)

$$
\begin{aligned}
l x^{\prime}+m y^{\prime} & =2 \text { quad. OLPM, } \\
\text { and }-n & =2 \text { quad. OLQM. } \\
\therefore \quad l x^{\prime}+m y^{\prime}+n & =2 \text { quad. LQMP, } \\
& =L M . P Q . \\
& =P Q \sqrt{l^{2}+m^{2}} . \\
\therefore \quad P Q & =\frac{l x^{\prime}+m y^{\prime}+n}{\sqrt{l^{2}+m^{2}}} .
\end{aligned}
$$

It is evident that if $P$ lies on the origin side of $l x+m y+n=0$, then quadrilateral $O L P M$ will be less than quadrilateral $O L Q M$, so that we shall have 2 quad. $L Q M P=-\left(l x^{\prime}+m y^{\prime}+n\right)$. It follows therefore that the line $l x+m y+n=0$ divides the plane of the axes into two regions, such that the expression $l x+m y+n$ is positive for points on one side of the line, and negative for points on the other. 
MATHEMATICAL NOTES.

II. The equation $l x+m y+n z+u=0$.

Take $L$ on $O X, M$ on $O Y$, and $N$ on $O Z$, so that $O M . O N=l$, $O N . O L=m$, $O L . O M=n$.

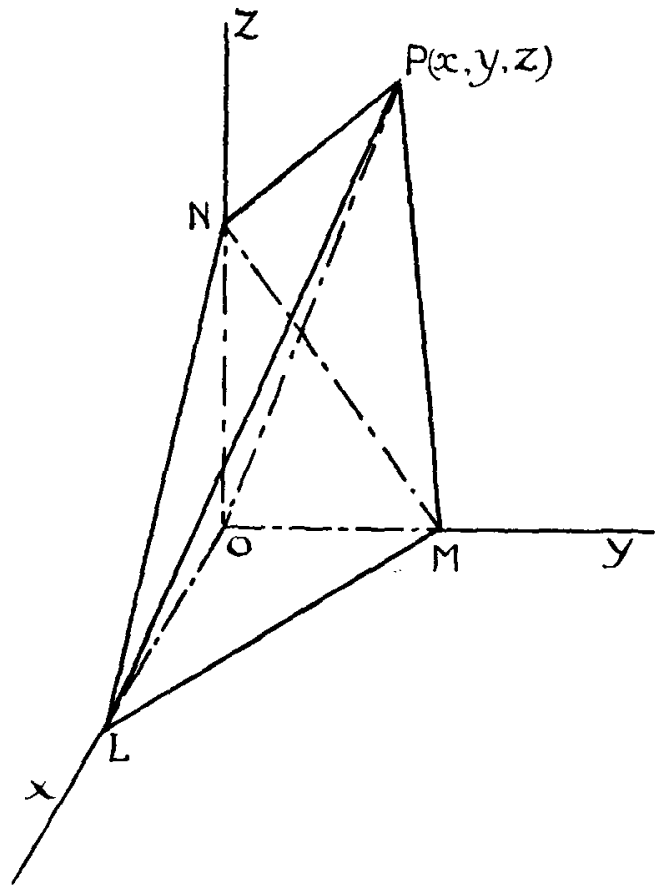

Let $P \equiv(x, y, z)$.

Then tet.hd. $(P O M N)+$ tet.hd. $(P O N L)+$ tet.hd. $($ POLM $)$ $=$ solid $O L M N P$, $\therefore \quad \frac{(O M \cdot O N) x}{6}+\frac{(O N \cdot O L) y}{6}+\frac{(O L . O M) z}{6}=$ solid OLMNP, $\therefore \quad l x+m y+n z=6$ solid $O L M N P$.

Hence if $P$ lie on the locus $l x+m y+n z+u=0$, then solid $O L M N P=-\frac{u}{6}$, $=$ constant volume. 
Take away the fixed part tet.hd. OLMN, $\therefore$ tet.hd. $(P L M N)=$ constant volume.

But $\triangle L M N$, the base of the tetrahedron is fixed, so it follows that the locus of $P$ is a unique (in virtue of volume of solid $O L M N P$ ) plane parallel to $L M N$.

Corollaries similar to those found in the case of the two dimensional equation can be deduced for the three dimensional one, and the length of the perpendicular from a point to a plane can be obtained by a method similar to (ii) of I.

John Milne.

\section{A simple form of Integrometer.}

Most continuously recording instruments are arranged so as to draw a graph exhibiting the quantity measured by means of cartesian coordinates. The record is on a roll of paper unwound from a drum. If the instrument be some form of meter, the value of the output, represented by $\int y d x$, may be obtained by measuring the area by a planimeter. The records, however, are somewhat bulky, and in this respect a polar graph is preferable. This is drawn by an indicator moving radially on a revolving disc. The peak or maximum of the curve is usually the most important, and this is the part which is represented best on such a polar diagram. Space may be further economised by having more than one convolution on one sheet of paper. Polar output records, however, are not freely used, perhaps because the planimeter does not give directly what is wanted, as it measures $\frac{1}{2} \int r^{2} d \theta$, instead of $\int r d \theta$.

The "Universal Planimeter" manufactured by A. Ott, and described in "Modern Mathematical Instruments" (G. Bell \& Sons) may be used as a radial integrator, and a method of adapting the ordinary planimeter to this case is given by Russell and Powles in the Engineer of January 1896.

A different form of integrometer has been made by the writer.

Let $O$ be the pole and $P$ the point $(r, \theta)$, and let $P$ trace a polar graph. Then if a rod $O P$ turn about $O$, and if the point of 